\title{
FACTORES DETERMINANTES DEL SANGRAMIENTO INTRA-OPERATORIO EN AMIGdALECTOMÍA PEDIÁTRICA
}

\author{
Ojeda Dagoberto ${ }^{1}$, Carranza Isabel $^{2}$, Rubio Mariluz $^{1}$, Agurto Mariela $^{1}$, Cisternas Patricia $^{3}$ \\ Anestesiólogo Clínica Dávila. \\ Otorrinolaringóloga Clínica Dávila. \\ Jefatura Anestesiología Clínica Dávila.
}

Introducción: La amigdalectomía y/o adenoidectomía es la cirugía más común en niños ${ }^{1}$. En la evaluación preoperatoria de estos pacientes es capital identificar a aquellos con riesgo aumentado de sangramiento, a este respecto se confiere mayor importancia en la actualidad a los antecedentes anamnésticos que a los exámenes de coagulación ${ }^{2}$.

Objetivo General: Identificar las variables que aumenten el nivel de sangramiento intraoperatorio en cirugía de amigdalectomía y/o adenoidectomía en pacientes pediátricos.

Material y Métodos: Se realizó un estudio observacional prospectivo desde noviembre de 2015 hasta agosto de 2016 en pacientes pediátricos sometidos a amigdalectomía y/o adenoidectomía electivas; se consignaron características demográficas basales, exámenes de coagulación, técnica anestésica, drogas y técnica quirúrgica. El sangramiento intraoperatorio se midió restando al contenido del frasco de aspiración la cantidad de suero fisiológico utilizado y dada la variabilidad en el tamaño de los pacientes se cuantifico en $\mathrm{ml} / \mathrm{kg}$ de peso. El sangramiento intraoperatorio no se distribuia en forma normal por lo que el análisis estadístico se realizó utilizando regresión cuartílica en forma univariada primariamente y luego se seleccionaron las variables independientes con un $\mathrm{p}$-valor $\leq 0,2$ para conformar un modelo multivariado. El software utlizado fue STATA 13.

Resultados: Se obtuvo información de 429 pacientes (edad mediana de 4 años, mín.-máx.; 1-14), con leve predominio masculino, mayoritariamente ASA 1, los resultados de protrombina, ttpk, recuento plaquetario y tiempo de sangría fueron en promedio normales. $2,4 \%$ de los pacientes requirieron evaluación hematológica. $\mathrm{La}$ inducción anestésica fue inhalatoria en su mayoría con sevoflurano, la técnica quirúrgica fue principalmente con disección (asa o Daniels) y la hemostasia fue predominantemente con subgalato, ligadura o puntos. Se utilizó Ketoprofeno mayoritariamente y $5 \%$ de los pacientes requirieron Ácido Tranexámico. La mediana del sangramiento intraoperatorio fue de $0,95 \mathrm{ml} / \mathrm{kg}$ $\mathrm{y}$ este fue influenciado por el tiempo quirúrgico (aumento de $0,05 \mathrm{ml}$ sobre la mediana por cada minuto), por el uso de mayores dosis de Ketoprofeno $(1,1 \mathrm{ml}$ por cada $\mathrm{mg} / \mathrm{kg})$ y los pacientes ASA 2 tuvieron $0,53 \mathrm{ml}$ sobre la mediana del sangramiento en relación a los ASA 1.

Conclusiones: El sangramiento intraoperatorio en cirugía de amigdalectomía pediátrica aumentó en relación a la duración de la cirugía, al grado de la clasificación de ASA y marcadamente por el uso de dosis mayores de Ketoprofeno. Destacó la nula influencia del nivel de los exámenes de coagulación, de la técnica quirúrgica, del uso de procoagulantes o de la presencia de enfermedades de la coagulación.

\section{Referencias}

1. Becke K. Anesthesia for ORL surgery in children. Head \& Neck Surgery 2014; 13.1-16.

2. Zaher G, Al-Noury K. The value of routine preoperative testing in the prediction of operative hemorrhage in adenotonsillectomy. Indian J Otolaryng Head Neck Surg 2014; 66:S30-S36. 